\title{
SVM-Based Real-Time Identification Model of Dangerous Traffic Stream State
}

\author{
Ming Huang (iD \\ School Wuhan University of Technology, Wuhan, Hubei 430063, China \\ Correspondence should be addressed to Ming Huang; hm@whut.edu.cn
}

Received 30 December 2021; Revised 17 January 2022; Accepted 18 January 2022; Published 11 February 2022

Academic Editor: Xin Ning

Copyright ( $) 2022$ Ming Huang. This is an open access article distributed under the Creative Commons Attribution License, which permits unrestricted use, distribution, and reproduction in any medium, provided the original work is properly cited.

By comparing and studying the correlation between traffic stream parameters and traffic safety of different highways, the correlations of traffic natural quantity, traffic equivalent, passenger-cargo ratio, car following percentage, congestion degree, and time occupancy rate are obtained. The traffic stream state before the actual accident is used as the criterion to judge the bad traffic stream state. The main parameters are obtained by extracting the parameters from the traffic stream data at the lane level and reducing the dimension of the parameters with the principal component analysis method. Establish a SVM model for RT early warning of traffic stream safety. Compared with other methods, the adaptive parameter selection method can adaptively select parameters according to the training sample set, realize the adaptive ability of the forecast model, and effectively improve the forecast accuracy of traffic stream. This paper studies the risk early warning model of road traffic accidents, which can transform the problem of road traffic safety into active early warning and improve the level of traffic safety. This study provides safety management measures for highway operation departments, which has certain theoretical significance and practical application value.

\section{Introduction}

With the rapid development of social economy, the number of cars is increasing linearly, and the increasing number of private cars not only is convenient for citizens to travel, but also brings about problems such as traffic congestion and environmental pollution [1]. Due to the limited resources of roads, many vehicles have a lot of traffic violations because of their own time, which brings potential traffic accident risks [2]. Reducing the occurrence of traffic accidents is the main task faced by highway operators for a long time. At present, only the prevention of traffic accidents can not meet the actual and future highway development requirements [3]. For now, with the rapid development of intelligent transportation system, the automatic identification of vehicle movements by traffic video has become a hot spot [4]. Because traffic video contains parameters such as traffic stream, vehicle behavior track, and vehicle license plate, it can be used to predict and identify vehicle behavior and traffic events [5]. Because of the strong nonlinear, random, and time-varying characteristics of the urban traffic system itself, the traditional forecasting methods based on accurate mathematical models are not ideal, so people pay more and more attention to AI $[6,7]$. Intelligent surveillance video can more accurately and effectively identify vehicle behavior, so as to solve the problem that traffic managers need to manually process a large number of video sources and realize low labor force and efficient detection, identification, and alarm.

Highways have played a significant role in promoting national economic growth and social development, thanks to the continuous improvement and encryption of China's highway network. Urban road traffic state recognition analyzes various traffic data using various discrimination algorithms, compares the results to prior traffic state standards, determines the current traffic system operation state, and implements intelligent traffic control, management, and guidance based on the discrimination results. The smoothness and safety of an urban expressway, as an important part of the urban road network, will have a direct 
impact on the smooth, orderly, and safe operation of the entire road network [8]. As a result, the key to effectively improving modern urban dangerous traffic streams is to analyze urban expressway traffic streams using various identification algorithms based on real-time (RT) traffic data collection [9]. At the moment, urban road traffic state classification and recognition research is primarily focused on two aspects. 1. The classification and analysis of RT Traffic data is the focus of the road traffic status classification. 2. The study of traffic state recognition methods based on a priori classification is the focus of traffic state recognition research. This paper summarizes the current state of traffic stream forecast models research, compares the benefits and drawbacks of forecast models, and examines the relationship between traffic stream data and the major factors influencing traffic stream changes. A Support Vector Machine (SVM) based RT recognition model of a dangerous traffic stream state is built.

The purpose of traffic guidance is to provide travelers with the best way to travel in the traffic network [10]. Traffic stream forecast and traffic state discrimination are two key problems in traffic guidance system. Providing RT and accurate traffic stream information and traffic state information can provide reference for travelers to make reasonable route selection [11]. Realizing fast and effective traffic state recognition is an important guarantee for realizing RT intelligent and effective control of urban traffic [12]. It is the goal of this paper to introduce the idea of active early warning control into traffic safety management, build a RT and efficient early warning model of traffic accident risk, reduce the occurrence of highway traffic accidents, and provide safe and efficient experience for highway travelers. By dividing the traffic parameter data of urban expressway and identifying its traffic state, it can help the traffic management department to grasp the running state of the whole traffic system in real time [13]. It is also the premise to ensure RT monitoring of urban expressway and intelligent traffic control in cities. Based on the influence of traffic stream on traffic safety, this paper fully excavates the potential laws and characteristics between traffic accidents and traffic stream characteristics of expressway and constructs a RT Traffic accident risk early warning model based on traffic stream, which provides safety management measures for expressway traffic management.

\section{Related Work}

A nonlinear, cycle-based traffic classification method based on traffic network state was proposed in the literature [14]. This nonlinear method has good stability and is not easily affected by dynamic traffic port changes. The improved algorithm was applied to traffic stream forecasting in literature [15], and it was compared to traditional multiple linear regression and SVM methods. The improved method has a higher forecast accuracy, according to the findings. By analyzing the macroscopic dynamic traffic stream model of an expressway and studying the selection of SVM parameters, literature [16] proposes an SVM forecast model for expressway dynamic traffic stream. The interaction between upstream and downstream traffic conditions was considered in literature [17], and a short-term forecast model based on SVM regression machine for road traffic status was established. The literature [18] employs the principal component analysis method and least squares SVM regression to forecast traffic streams from multiple sections as a whole. As an example, cross section data from an urban expressway was used for verification. The problem of RT Traffic signal recognition was addressed in literature [19], which used video to analyze the specific identification indicators of the image to identify and analyze the traffic state. Based on traffic stream and nonlinear theory, literature [20] proposed an expressway traffic state recognition method. An urban road traffic abnormal event management system was designed in the literature $[21,22]$. The system created a system data flow diagram, described the model division of the system, and described the method of refining each module by creating a logic tree for each module based on the functions that should be realized. Finally, the system's network architecture is presented. Literature [23] is based on the basic data collected by the traffic detector on the roads, which is then analyzed and processed using traffic engineering theory and data mining techniques to determine the respective characteristics of these roads from their basic traffic data. Finally, by the cluster analysis method, according to the certain similarity of the sample flow, speed, and occupancy rate of each traffic state and the difference of the samples between different states, a reasonable traffic state classification suitable for different roads is obtained. Literature [24] proposed an automatic identification method of traffic congestion based on SVM, which can identify the type of traffic congestion. Simulation shows that this algorithm has a higher detection rate and a lower false alarm rate than traditional detection methods. Literature [25] proposed a traffic stream time series forecasting method based on statistical learning theory and proved the effectiveness and advancement of the method through experiments, discussed the application of SVM in traffic stream forecast, and compared it with neural network. Literature [26] adopts the sequential minimum optimization algorithm. When the input signal is mixed with noise, SVM is more robust, and the forecast results are better than neural networks and dynamic recurrent neural networks. Literature [27] uses the nuclear machine method to predict the traffic stream in real time and compares it with the SVM method. Literature [28] proposed a short-term traffic stream forecast model based on SVM. The model is superior to the forecast model based on neural network in terms of accuracy, convergence time, generalization ability, and optimality.

The article researches on SVM parallel computing technology. For the dynamic traffic stream forecast of largescale road networks, parallel computing is an important way to quickly train large sample sets in short-term traffic stream forecast. Based on the research of parallel technology, parallel algorithms are introduced for traffic stream forecasting, and parallel experiments are conducted on multiple road sections at the same time. Through simulation experiments, the finally constructed SVM model can successfully identify the bad traffic stream state corresponding 
to most accidents and can effectively monitor and warn the bad traffic stream state on the highway in real time. The practicability and usability of the forecast of this model are verified.

\section{Methodology}

3.1. Traffic Stream Forecast Analysis. Traffic stream is a holistic and macroscopic concept. Through the analysis of a large number of observation data, it is found that traffic stream has a certain characteristic tendency, so the concept of traffic stream characteristics is put forward [29]. Traffic stream characteristics refer to the qualitative and quantitative characteristics of traffic stream operation state. There are many methods to distinguish traffic stream state, from simple manual patrol to emergency telephone and mobile telephone. From CCTV discrimination to automatic electronic surveillance discrimination, etc, these methods can find the traffic congestion on the road to a certain extent and within a certain range. For now, the development of video image processing technology has created conditions for improving the effectiveness of direct traffic stream state discrimination algorithm.

The characteristics and characteristics of fluid are shared by a large number of pedestrians and vehicles passing through the road. People and vehicles passing through a road are collectively referred to as a traffic stream in traffic engineering. In traffic engineering, the term "traffic stream" usually refers to vehicle flow. The premise of intelligent traffic guidance systems and traveler information release systems [30] is RT and accurate traffic stream information, and traffic stream forecast is an important aspect of intelligent transportation systems. The only way to distribute the road load reasonably, provide a reference for travelers to travel, and solve the problem of road traffic congestion is to have traffic stream information.

It is necessary to analyze the correlation influence of traffic stream parameters on traffic safety before studying the traffic accident early warning model based on traffic stream parameters. The road will not change after the route design is completed, and the impact on traffic safety is certain, among the factors affecting expressway traffic safety. Driver factors, vehicle factors, and other environmental factors, on the other hand, have RT changing characteristics, which will eventually manifest in changes in traffic stream characteristics, affecting highway traffic safety. Microparameters describe the operating state and characteristics of the traffic stream as a whole, whereas macroparameters describe the operating state and characteristics of the traffic stream as a whole.

Under normal circumstances, the traffic stream is large, and the traffic density is also large. However, when the road traffic is very crowded, and the traffic stream is stagnant, the traffic stream is approximately equal to zero, but the traffic density at this time is close to the maximum. Therefore, it is difficult to express the actual state of traffic stream simply by using traffic stream indicators, and better evaluation can be made by using traffic density indicators. Usually, there are abnormal data in traffic detection data due to hardware equipment failure, noise interference, and other factors. Therefore, it is necessary to eliminate the wrong data; otherwise, the abnormal data will affect the model results. Considering that the abnormal data is caused by equipment failure and noise interference, it is quite different from normal data and does not meet certain rules. Therefore, threshold method and logical inference rules are used to eliminate abnormal data. The regression forecast flow based on SVM in this paper is shown in Figure 1.

The space occupancy rate on a given road section is the ratio of the total length of vehicles to the total length of the road section, which is usually expressed as a percentage. The level of traffic density is directly reflected in the space occupancy rate, but it can better show the actual occupation of roads. Because it is difficult to obtain traffic parameter data directly, similar to traffic density, it is rarely used in practice. The goal of traffic stream characteristics research is to describe and explain the fundamental properties and rules that govern traffic flow state. The study of expressway traffic stream characteristics provides a solid foundation for examining the relationship between traffic stream parameters and traffic safety. The relationship between any two basic traffic stream parameters, such as traffic volume, speed, and density, is studied by traffic stream characteristics.

Changes in traffic stream parameter data can reflect temporal and spatial changes in traffic stream characteristics. The traffic stream state can be monitored by analyzing the change law of these traffic data if a certain number and type of traffic detectors are installed on the road, and the traffic parameter data is sampled at regular intervals. Traffic stream characteristics differ in space; that is, they differ in different locations affected by weather, visibility, and road alignment conditions, but they are similar in different sections of the same road section. The change of traffic stream is affected by both frequent and occasional road congestion. As a result, the factors that influence traffic flow change do not exist in isolation, but rather interact and influence one another, resulting in a complex dynamic process. The interaction relationship is depicted in Figure 2.

As the most basic variable, traffic parameters are related to the rationality and accuracy of the final traffic state discrimination, and also to the operability and portability of the design algorithm. Therefore, the selection of traffic parameters must follow several principles. (1) intuitive principle: the adopted traffic parameters should be easy to understand. (2) practical principles: the adopted traffic parameters should meet the needs of the commonly used traffic state discrimination algorithms. (3) Primitiveness principle: the traffic parameter data to be used as input variables should be obtained directly by the existing conventional traffic detection technology, or by simple calculation. (4) Principle of economy: the adopted traffic parameters should have good economy when obtaining.

Generally speaking, every traffic accident is uncertain. That is, traffic accidents of different severity may occur in any place, any time, and any environment. However, when the number of traffic accidents increases, mathematical statistics on the macro level will show certain characteristics and laws. Establishing the correspondence between traffic 

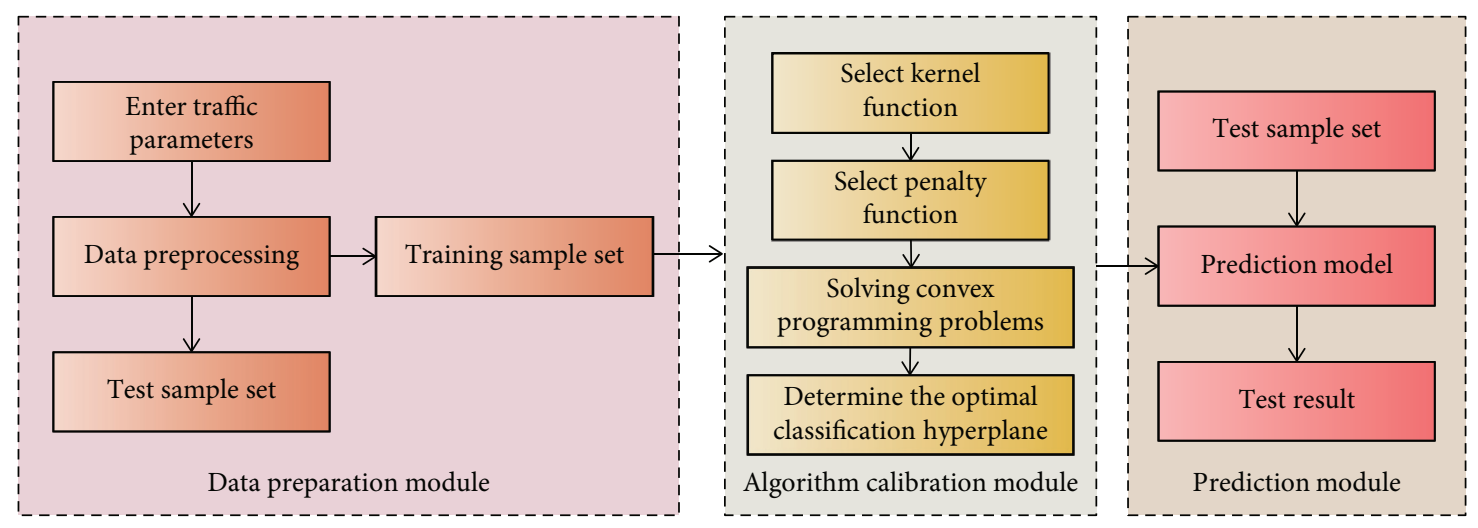

FIGURE 1: SVM regression forecasting process.

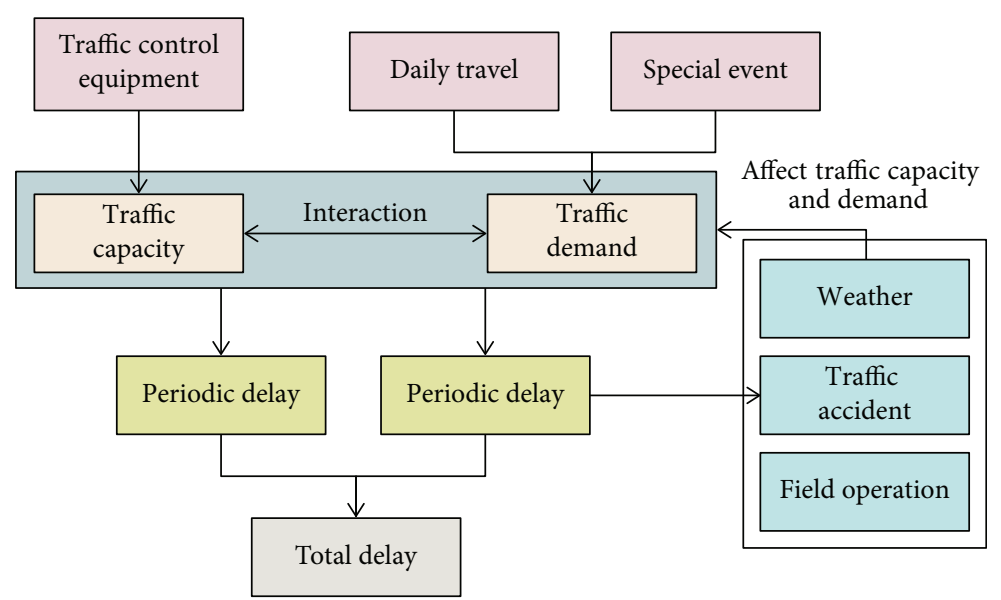

Figure 2: Traffic congestion analysis.

accidents and time, it is impossible to effectively judge the traffic safety status of expressway only by the number of accidents. Therefore, this study adopts the "accident equivalent method"; that is, the traffic accident rate is used to establish the correlation between traffic stream parameters and traffic safety.

\subsection{SVM Based Traffic Stream RT Early Warning Model.} In the research of traffic engineering, the average driving speed, road share, and average traffic stream are important parameters to reflect the characteristics of traffic stream. In different areas such as urban road sections, intersections, and road networks, the traffic stream in a specific time period shows different characteristics in these parameters. In this paper, three characteristic parameters of road traffic parameters, namely, traffic stream, average speed, and occupancy rate, are selected, and the corresponding calculation formulas are appropriately revised to meet the parameter settings of the simulation system, so as to build a threedimensional sample space. Time average speed reflects the running status of traffic stream at a specific observation site, and interval average speed reflects the running status of traffic stream at a specific road section. Both time average speed and interval average speed are important indicators in traffic management and control, which are of great significance to traffic planning, road design, vehicle operation efficiency, traffic management and control, etc.

There are many decisive factors in choosing traffic parameters when judging the RT Traffic state. For example, it is necessary to consider the awareness of traffic participants, the current status of equipment, the basic requirements of traffic state discrimination algorithm, etc. Due to the missing data of the detector or the fault status information returned by the detector coil in the partial period of the original data, the data returned by the database query will be null or invalid. Accidents with abnormal data should be ruled out. Similarly, there will be data missing, null return, and invalid data in the data query of the control group detector, which needs to be excluded. The kernel function $k$ of SVM model mainly includes the following:

Linear kernel function:

$$
K\left(x, x_{i}\right)=x^{T} x_{i}
$$

Polynomial kernel function:

$$
K\left(x, x_{i}\right)=\left(\gamma x^{T} x_{i}+r\right)^{p}, \quad \gamma>0 .
$$

Radial basis kernel function: 


$$
K\left(x, x_{i}\right)=\exp \left(-\gamma\left\|x-x_{i}\right\|^{2}\right), \quad \gamma>0 .
$$

Two-layer perceptron kernel function:

$$
K\left(x, x_{i}\right)=\tanh \left(\gamma x^{T} x_{i}+r\right) .
$$

Data standardization is a term that refers to the process of standardizing data. The data of different variables are different in order of magnitude in practice. Data normalization is used to transform the data of different variables into numbers between $[0,1]$ in order to avoid large errors in the network model caused by differences in order of magnitude. Because the results will be influenced by the different values of various traffic stream parameters, it is necessary to standardize the traffic stream parameters.

Different models differ in terms of size and dynamic performance. Large cars, in most cases, are unable to maintain a constant following distance with small cars. As a result, a disjointed motorcade is created. The normal traffic flow is affected by the changing and unstable traffic composition. Furthermore, large cars will block the line of sight of vehicles following them, posing a hidden risk of traffic accidents. As a result, research into the relationship between the passenger-to-cargo ratio of traffic vehicles and the rate of traffic accidents is required. The observation matrix is defined as [density, flow] in the process of determining the state of urban traffic. To achieve the classification of traffic congestion and free state, select the appropriate kernel function and add the observation matrix to the SVM discriminant function. SVM is proposed in the optimal classification under the condition of linear separability. Its classifier is

$$
f(x)=\operatorname{sgn}(w x+b)=\operatorname{sgn}\left(\sum_{i=1}^{l} \alpha_{i}^{*} y_{i}\left(x_{i} x_{j}\right)+b^{*}\right) .
$$

Among them, $\alpha_{i}$ is the optimized solution of the quadratic problem.

$$
\begin{aligned}
& \min _{\alpha} \frac{1}{2} \sum_{i=1}^{l} \sum_{i=1}^{l} y_{i} y_{j} \alpha_{i} \alpha_{j}\left(x_{i} x_{j}\right)-\sum_{j=1}^{l} \alpha_{j}, \\
& \text { Subjected to } \sum_{i=1}^{l} y_{i} \alpha_{i}=0 .
\end{aligned}
$$

In formula (5), $b$ can be obtained by establishing the following equation (support vector), $0 \leq \alpha_{i} \leq C, \quad i=$ $1, \ldots, l$.

$$
y_{i}\left[\sum_{i=1}^{n} \alpha_{i}\left(x_{i} x\right)+b\right]-1=0 .
$$

Under the condition of inseparable linearity, nonlinear conversion is realized through SVM kernel function. The kernel function is expressed as

$$
K\left(x_{i}, x_{j}\right)=\left(\phi\left(x_{i}\right) \phi\left(x_{j}\right)\right) .
$$

From the expression form of kernel function, the proper type of kernel function and the choice of kernel function parameters have an important influence on the construction of matrix, and the smoothing accuracy and the complexity of the model can be weighed.

The state of a road traffic stream can be used to describe its characteristics. Because of the interaction between traffic demand, vehicle operation characteristics, and road facility service capacity, traffic streams have different characteristics depending on their state. The traffic stream is divided into three states in this paper: free flow, crowded flow, and blocked flow. The ratio of the sum of the vehicle lengths on the observed road section to the total length of the road section in a given time period is known as the space occupancy rate. The space occupancy rate is a direct reflection of traffic density and can provide a more accurate picture of the road's actual use. However, obtaining this traffic parameter directly is difficult. The traffic congestion identification method of urban expressway based on nonlinear classification method is an important research direction that focuses on multiparameter measured data. It is proposed to create a two-dimensional space for a traffic stream-density model, as well as an SVM classification method for traffic states of noncongestion flow and congestion flow on an urban expressway.

\section{Result Analysis and Discussion}

For the participants surveyed by all parties, the recognition of vehicle speed in traffic state discrimination is higher, which also shows that vehicle speed can most intuitively reflect the running state of traffic stream. Secondly, the usage ratio and recommended usage ratio of the two basic traffic parameters, traffic stream and occupancy rate, are also significantly larger than the other remaining parameters. In the data type, the flow rate, speed, and density returned by the detector are adopted. In the course of the experiment, the total sample data is divided into different proportion sample sets by means of cross-validation, and the comparative experimental analysis is carried out. In the cross-validation group, this study is divided according to the proportion of different training sets, validation sets, and test sets.

On the real road, we collect data on vehicles driving straight ahead, overtaking, and changing lanes. Except for slight differences in slopes, the majority of the vehicle's motion fits straight lines. Overtaking on the left and right is distinguished by their relatively large curvature, and when the vehicle completes overtaking, the fitting slope of the final driving track segment returning to the original lane maintains a small difference with the slope of the starting straightahead segment. Gather traffic video on the actual road, track the vehicles in the video to obtain the RT running track, and cluster the tracks using least square piecewise fitting and fuzzy C-means. The clustering effect is shown in Figure 3.

Traffic congestion is the key factor that directly affects drivers' psychological changes. When the traffic congestion is small, the interaction between vehicles is small, which easily leads to dangerous driving behaviors at superhigh 


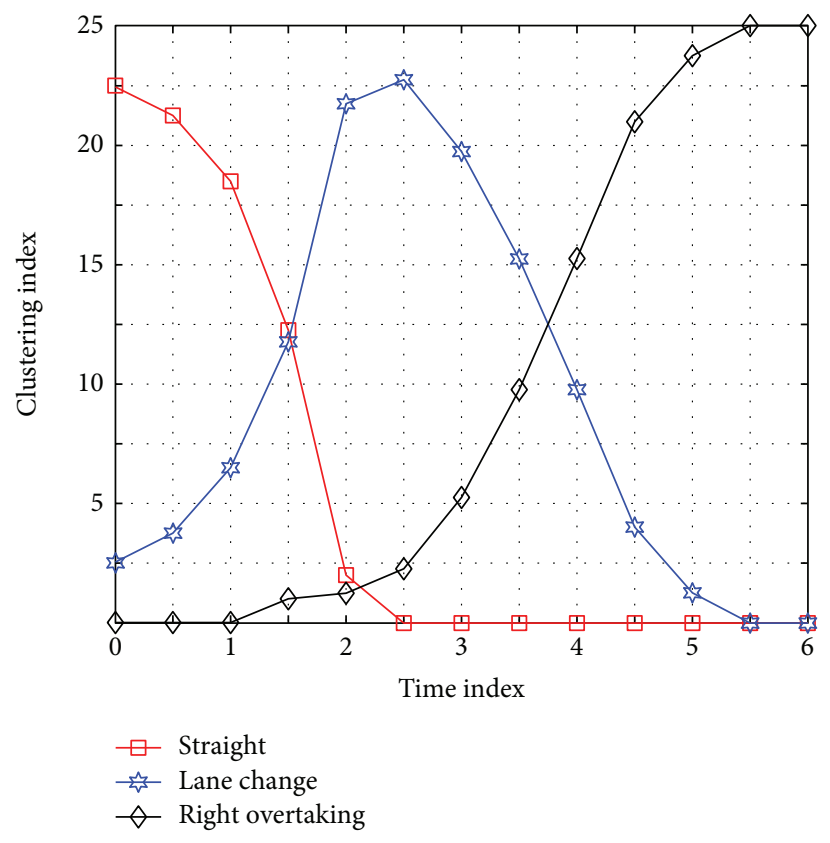

Figure 3: Clustering effect map.

speed. In case of accidents, emergency and effective measures cannot be taken, resulting in traffic accidents. When the traffic congestion is high, the driver's psychological tension increases, which leads to improper operation of drivers and traffic accidents.

Using the trained SVM model, the state of the test samples is judged, and the test samples are the traffic stream parameters of one hour in the research range. The model test results are shown in Figure 4. Among all 300 samples, the classification accuracy rate is $92.22 \%$. Therefore, only 24 hours of data can be used to train the model that can meet the traffic stream state discrimination.

The model is built on the foundation of useful data. The identification of traffic operation state in this study will require completing data extraction, fusion, preprocessing, and other processes based on historical traffic accident data and traffic stream data. Traffic accidents are caused by changes in the traffic stream, and the traffic stream parameters of traffic accident locations can be obtained during data extraction. Some invalid data is removed, and data from the traffic stream is converted after data fusion.

The influence of vehicle overspeed on braking is mainly manifested in braking acceleration and braking distance. This study mainly analyzes the influence of overspeed on braking distance. According to the experience of different vehicle types, the braking distance and the influence of vehicle speed on braking are shown in Figure 5.

The case matching control method, which is commonly used in epidemiology, is used for sample selection in order to effectively distinguish the bad traffic stream states, which means that several groups of normal traffic stream state data corresponding to the bad traffic stream states corresponding to valid accident samples must be extracted as the control group. The method of mathematical statistics is commonly used in mathematical models that describe the change in driving risk value. The data of traffic detection equipment in traffic accident-prone road sections throughout the city was collected based on existing data, and the overall distribution density was estimated using the parameter estimation method. The maximum likelihood parameter estimation method is used to fit distribution functions in this paper, and the fitting effect is evaluated using maximum similarity. MATLAB is used to carry out the fitting process, and the total amount of data collected is 3000 . The fitting results are shown in Figure 6.

It can be found that the greater the similarity, the better the fitting effect. The normalization of data has an important influence on SVM classification. By comparing the classification results of normalized data and nonnormalized data under different kernel functions, it can be seen that, under the same parameter setting conditions, kernel function and Sigmoid kernel function have good classification effect on normalized data, while polynomial kernel function has obvious classification effect on nonnormalized data. In this paper, empirical method, particle swarm optimization method, and adaptive parameter selection method are used to select SVM penalty parameters and insensitivity coefficients. The forecast result of adaptive parameter selection is shown in Figure 7.

It can be seen that the adaptive parameter selection method selects parameters according to samples and has the advantage of automatically selecting parameters. In practical application, human intervention is reduced, and in a certain sense, intelligent traffic stream forecast is realized, and its forecast accuracy can meet the demand of traffic stream forecast. In actual research work, only the first few principal components with the largest variance are usually selected, so as to simplify the system structure and grasp the essence of the problem. Compare the classification accuracy of each model, and the comparison results are shown in Figure 8. 


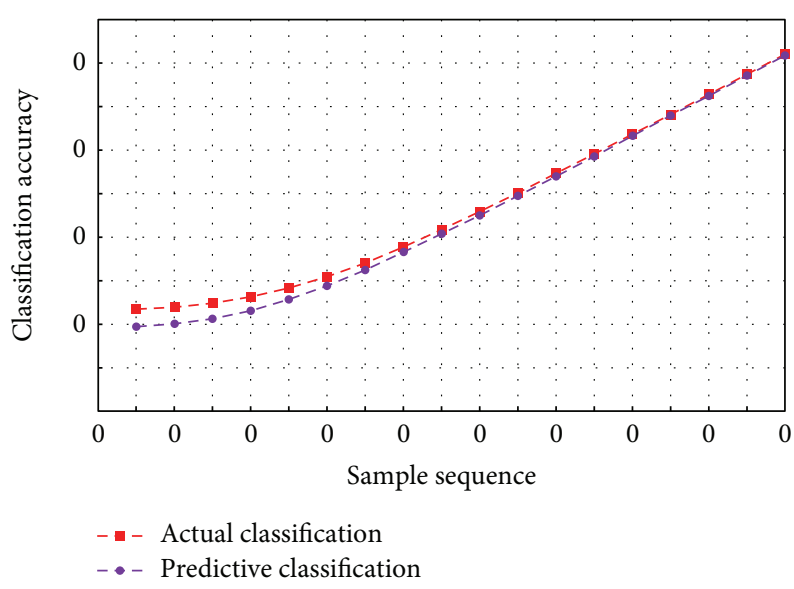

Figure 4: Test set sample classification result chart.

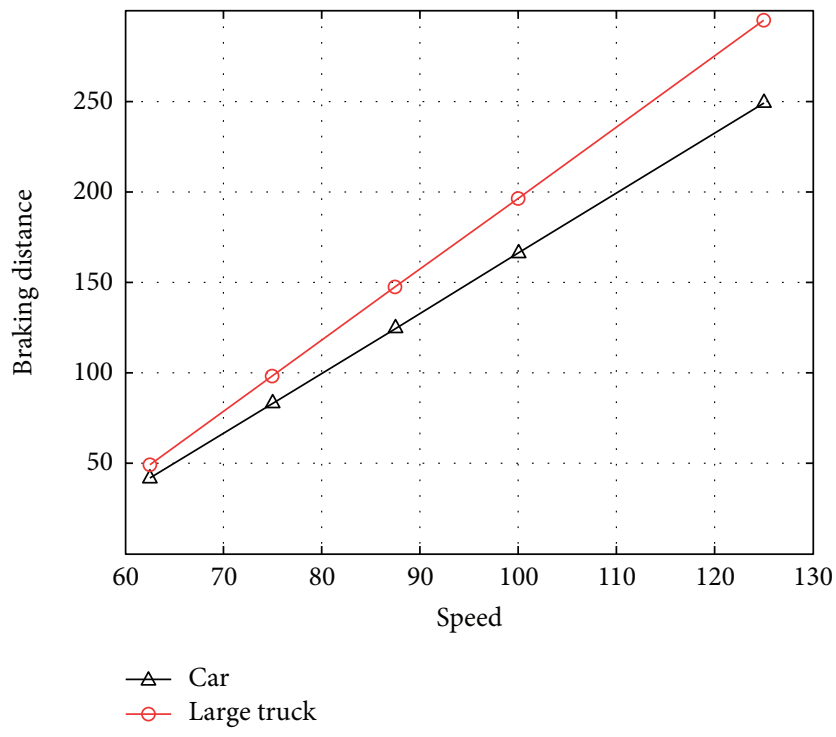

Figure 5: Influence of vehicle speed on braking distance.

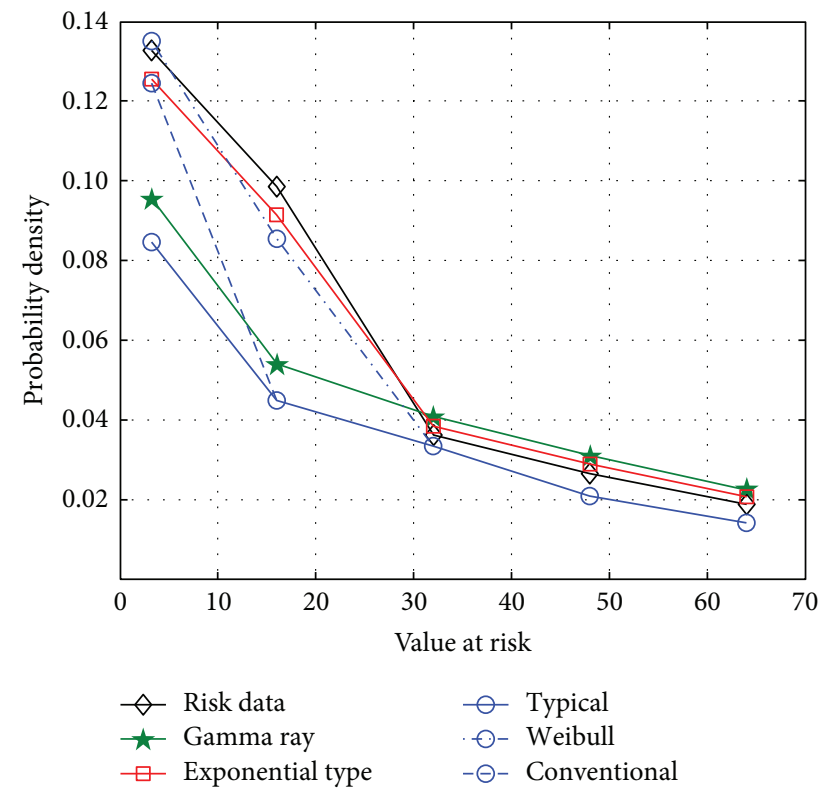

Figure 6: Comparison results of fitting of different distribution functions. 


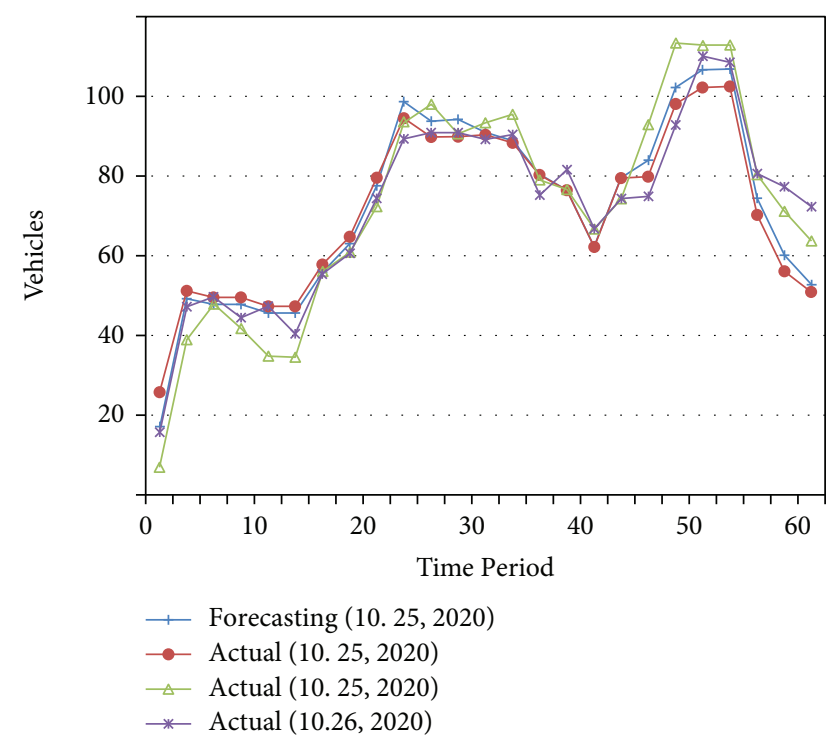

Figure 7: Traffic stream forecast based on adaptive parameter selection.

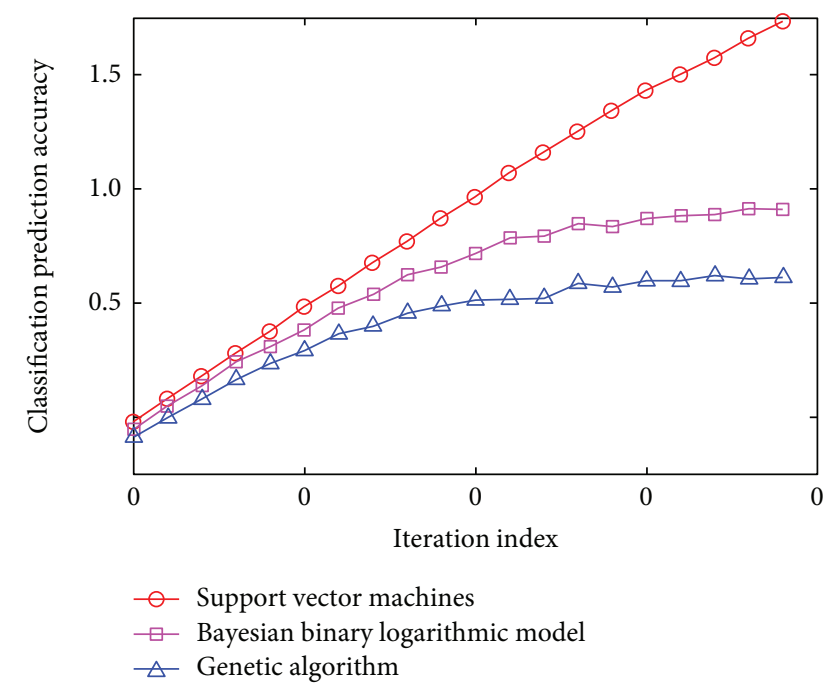

FIGURE 8: Comparison of model performance.

It can be seen that the RT monitoring algorithm proposed in this paper can effectively identify the bad traffic stream state, thus providing reliable decision support for traffic managers.

When the experimental results of the overall correct rate and accident correct rate in each group are compared, it is discovered that the experimental results in the training set are higher than those in the test set. It shows that, during the experiment, the phenomenon of "overfitting" occurs, and that as the number of training set samples and the proportion of verification set data samples decrease, the phenomenon of "overfitting" weakens. It was discovered that, under the three indexes of single lane flow, average speed, and occupancy rate, and four classification labels of congestion flow, congestion flow, steady flow, and smooth flow, whether to process data or not, and the classification results of SVM kernel functions, all types of kernel functions can have high classification accuracy. It demonstrates that classifying with this classification label and index has a positive application effect.

\section{Conclusions}

With the vigorous development of the world economy and the rapid development of the transportation industry, on the one hand, it promotes the exchange of materials and people, greatly shortens the travel time, and then improves the work efficiency. On the other hand, it also brings many disadvantages. Based on the existing data, the data of traffic detection equipment in traffic accident-prone road sections around the city were collected, and the overall distribution density was estimated by parameter estimation method. Based on the influence of traffic stream on traffic safety, this paper fully excavates the potential laws and characteristics between traffic accidents and traffic stream characteristics. A SVM based RT identification of dangerous traffic stream state is proposed. This paper adopts the technical route of correlation analysis between traffic stream characteristics and traffic safety-traffic accident identification based on safety domain-traffic accident risk early warning model research based on traffic stream and applies related technologies such as traffic stream characteristics analysis, SVM, traffic safety analysis theory, principal component analysis technology, safety domain theory, early warning theory, and so on. The experimental results show that SVM model is used to realize RT monitoring and identification of bad traffic stream, and the accuracy of traffic accident identification model is improved by data preprocessing measures and can effectively identify the bad traffic stream state.

\section{Data Availability}

The data used to support the findings of this study are included within the article.

\section{Conflicts of Interest}

The author does not have any possible conflicts of interest.

\section{Acknowledgments}

This study was supported by the Construction of Intelligent Service System of QingDao Pilotstation (no.: 20213h511a).

\section{References}

[1] E. Y. Kim, "MRF model based real-time traffic flow prediction with support vector regression," Electronics Letters, vol. 53, no. 4, pp. 243-245, 2017.

[2] A. M. Kalteh, "Improving forecasting accuracy of streamflow time series using least squares support vector machine coupled with data-preprocessing techniques," Water Resources Management, vol. 30, no. 2, pp. 747-766, 2016.

[3] M. A. Ghorbani, H. A. Zadeh, and M. Isazadeh, "A comparative study of artificial neural network (MLP, RBF) and support vector machine models for river flow prediction," Environmental Earth Sciences, vol. 75, no. 6, pp. 1-14, 2016. 
[4] J. He, S. A. Mattis, T. D. Butler, and C. N. Dawson, "Datadriven uncertainty quantification for predictive flow and transport modeling using support vector machines," Computational Geosciences, vol. 23, no. 4, pp. 631-645, 2019.

[5] J. Sun and J. Sun, "Real-time crash prediction on urban expressways: identification of key variables and a hybrid support vector machine model," IET Intelligent Transport Systems, vol. 10, no. 5, pp. 331-337, 2016.

[6] J. Kong, H. Wang, X. Wang, X. Jin, X. Fang, and S. Lin, "Multi-stream hybrid architecture based on cross-level fusion strategy for fine-grained crop species recognition in precision agriculture," Computers and Electronics in Agriculture, vol. 185, Article ID 106134, 2021.

[7] X.-B. Jin, W.-Z. Zheng, J.-L. Kong et al., "Deep-learning temporal predictor via bidirectional self-attentive encoderdecoder framework for IOT-based environmental sensing in intelligent greenhouse," Agriculture, vol. 11, no. 8, p. 802, 2021.

[8] A. Bartolozzi, A. Soloperto, and G. Palazzolo, "Real-time identification of cell mechanical properties," Biophysical Journal, vol. 112, no. 3, p. 435, 2017.

[9] G. E. Sakr and I. H. Elhajj, "VC-based confidence and credibility for support vector machines," Soft Computing, vol. 20, no. 1, pp. 133-147, 2016.

[10] A. R. Rao, "Identification of donor splice sites using support vector machine: a computational approach based on positional, compositional and dependency features," Algorithms for Molecular Biology, vol. 11, no. 1, p. 16, 2016.

[11] Y. Zhu and Y. Zheng, "Retracted article: traffic identification and traffic analysis based on support vector machine," Neural Computing \& Applications, vol. 32, no. 7, pp. 1903-1911, 2020.

[12] M. Ogawa and H. Munetomo, "Wi-fi CSI-based outdoor human flow prediction using a support vector machine," Sensors, vol. 20, no. 7, p. 2141, 2020.

[13] Q. Ma, C. Sun, B. Cui, and X. Jin, "A novel model for anomaly detection in network traffic based on kernel support vector machine," Computers \& Security, vol. 104, no. 2, Article ID 102215, 2021.

[14] L. Guo, Q. Wu, and S. Liu, "Deep learning-based real-time VPN encrypted traffic identification methods," Journal of Real-Time Image Processing, vol. 17, no. 9, pp. 1-12, 2020.

[15] Z. Yang, T. Dong, A. Jensen, and E. Halvorsen, "Integratable capacitive sensor for identification of microfluidic two-phase flow patterns in lab-on-chip devices," Journal of Microelectromechanical Systems, vol. 25, no. 1, pp. 197-206, 2016.

[16] J. Lin, X. Lin, L. Guo et al., "Identification accuracy improvement for steel species using a least squares support vector machine and laser-induced breakdown spectroscopy," Journal of Analytical Atomic Spectrometry, vol. 33, no. 9, pp. 1545-1551, 2018.

[17] M. A. Ghorbani, R. Khatibi, and A. Goel, "Modeling river discharge time series using support vector machine and artificial neural networks," Environmental Earth Sciences, vol. 75, no. 8, pp. 1-13, 2016.

[18] X. Kong, Y. Sun, R. Su, and X. Shi, "Real-time eutrophication status evaluation of coastal waters using support vector machine with grid search algorithm," Marine Pollution Bulletin, vol. 119, no. 1, pp. 307-319, 2017.

[19] L. Azimi, M. Talebi, P. Owlia et al., "Tracing of false negative results in phenotypic methods for identification of carbapenemase by Real-time PCR," Gene, vol. 576, no. 1, pp. 166-170, 2016.

[20] M. A. Rahim, J. Liu, Z. Zhang, L. Zhu, X. Li, and S. Khan, "Who is driving? Event-Driven driver identification and impostor detection through support vector machine," IEEE Sensors Journal, vol. 20, no. 12, pp. 6552-6559, 2020.

[21] K. Li, Y. Wu, Y. Nan, P. Li, and Y. Li, "Hierarchical multi-class classification in multimodal spacecraft data using DNN and weighted support vector machine," Neurocomputing, vol. 259, no. 10, pp. 55-65, 2017.

[22] L. Huang, W. Ding, and W. Liu, "Identification of wheat powdery mildew using in-situ hyperspectral data and linear regression and support vector machines," Journal of Plant Pathology, vol. 101, no. 4, pp. 1-11, 2019.

[23] X. Qian, J. P. Chen, and L. J. Xiang, "A novel hybrid KPCA and SVM with PSO model for identifying debris flow hazard degree: a case study in Southwest China," Environmental Earth Sciences, vol. 75, no. 11, pp. 1-16, 2016.

[24] S. Dong, "Multi class SVM algorithm with active learning for network traffic classification," Expert Systems with Applications, vol. 176, no. 12, Article ID 114885, 2021.

[25] C. A. Tsiliyannis, "Real-time identification of mean retention time and end-of-life rate in cyclic manufacturing," Journal of Cleaner Production, vol. 140, no. 3, pp. 1553-1566, 2017.

[26] J. Guo, Z. Liu, W. Huang, Y. Wei, and J. Cao, "Short-term traffic flow prediction using fuzzy information granulation approach under different time intervals," IET Intelligent Transport Systems, vol. 12, no. 2, pp. 143-150, 2017.

[27] P.-S. Yu, T.-C. Yang, S.-Y. Chen, C.-M. Kuo, and H.-W. Tseng, "Comparison of random forests and support vector machine for real-time radar-derived rainfall forecasting," Journal of Hydrology, vol. 552, pp. 92-104, 2017.

[28] J. Lin, X. Lin, L. Guo, Y. Guo, and Y. Tang, "Identification accuracy improvement for steel species using a least squares support vector machine and laser-induced breakdown spectroscopy," Journal of Analytical Atomic Spectrometry, vol. 33, no. 9, pp. 1545-1551, 2018.

[29] S. Salehinia, A. Ghaffari, A. Khodayari, M. N. Khajavi, and F. Alimardani, "Modelling and controlling of car-following behavior in real traffic flow using ARMAX identification and model predictive control," International Journal of Automotive Technology, vol. 17, no. 3, pp. 535-547, 2016.

[30] M. Avvenuti, G. Cola, and A. Vecchio, "Real-time identification using gait pattern analysis on a standalone wearable accelerometer," Computer Journal, vol. 60, no. 8, pp. 1173-1186, 2017. 